\title{
Comparative studies on lumpy skin disease virus in human
}

\author{
Samia Ahmed Kamal* \\ Animal Health Research Institute (AHRI), Dokki, Giza, Egypt
}

\begin{abstract}
Background: Lumpy Skin Disease Virus (LSDV), capripoxvirus, family poxviridae, was recently (2018-2019- till present) exhibiting its characteristic zoonosis [animal to human]. Moreover, the person- to- person direct transmission [infectious disease] were recorded.

Materials and Methods: Whole blood and tissue samples from skin nodules were collected from infected persons (showing characteristic pox nodules). LSDV was isolated on BHK-21 cell culture, and pathogen free embryonated chicken eggs (CAM). Diagnosis were confirmed by conventional Polymerase Chain Reaction and partial sequencing using the primers target gene ORF103 and Negative staining, direct electron microscopic examination.

Results: LSDVh was successfully isolated from blood and skin nodules collected from infected persons. Confirmed diagnosis was achieved by the conventional PCR and sequencing procedures. The isolates were genetically characterized as Lumpy skin disease virus isolate Evros/GR/15 by partial sequence with $99 \%$ identity. Meanwhile, LSDV virions and herpes virus particles were visualized by electron microscopic investigations (NS-DEM). The majority of LSD virions were nonenveloped showing Mulberry forms and some capsular form, and the enveloped forms. Herpes virus virions were also detected in large number, the enveloped and the non- enveloped. The apparent pathogenesis on infected human are comparatively similar to LSD in cattle however the progress of illness and stages of the disease depends on level of health care interventions. Unless the nature of human biology is greatly different from that of cattle.
\end{abstract}

Conclusions: Lumpy skin disease virus is capable of infecting humans with direct transmission without the need for insects vector; that most probably by inhalation and certainly by the direct contacts with infected materials, infected persons [man to man], and as laboratory acquired infection. LSDVh causes skin nodules and may lead to death in cases of generalized infections and if involving the internal organs. However, it is obvious that Herpes virus gives hands and helping poxviruses during their pathogenesis in the infected subjects.

\begin{abstract}
Abbreviations: DEM: Direct electron microscope. LSDV; lumpy skin disease virus. LSDVh: lumpy skin disease in human, NS: negative staining. PCR, polymerase chain reaction. HHSV; human herpes simples virus. M form; Mulberry form. AHRI; animal health research institute.
\end{abstract}

\section{Background}

Lumpy skin disease virus (LSDV) of cattle belongs to genus Capripoxvirus [family poxviridae], double-stranded DNA. It causes outbreaks in Egypt that continues since the beginning of year 2018 till present 2019. LSDV have more than one form which are antigenically distinct [non-enveloped, enveloped, intracellular mature virus, cellassociated enveloped virus, extracellular enveloped virus, intracellular enveloped virus). It impairs the innate immune system, persist in nature in wild so that it is difficult to eradicate, moreover, vaccinations always fail to control recurrent infections in endemic countries. LSD discovered in cattle in Zambia in 1929. The first outbreaks appeared in Egypt in 1988, and the disease was first reported in the Europe in Cyprus in 2014, Greece in 2015, Balkan countries in 2016 Transmission between countries occurs through movements of infected cattle or through vectors in animal trucks. All ages and breeds of cattle are susceptible. The skin lumps in cattle either regress or progress to necrosis, ulcers and finally scars. Generalized infection can affect the respiratory- tracts, digestive system, genitalia and lymph nodes [1-7]. Negative stain EM images show that the surface membrane encloses a biconcave or cylindrical core that contains the genome DNA and proteins organized in a nucleoprotein complex. One or two lateral bodies appear to be present in the concave region between the core wall and a membrane. A recent model suggests that the nucleoprotein complex might be cylindrical, folded at least twice along the long virion axis to form a Z-structure, which presents as three circles, arranged linearly when viewed as a section across the short axis [8-10].

During the year 2018- 2019 a massive LSD enzootic was attacking Egypt, and sporadic human infections were recorded in Al Fayoum, Giza, Cairo, and Al Sharqia Governorates. Moreover, recently a laboratory acquired infection was reported (November 2019), and also in people by direct man to man transmission (November 2019). In this research the new findings focus on zoonosis of LSDV, the virus cross species barriers and the human infections are in progress and new infections were detected in persons of different communities. In human LSD apparently seen as a specific skin nodular disease, few mortalities were reported in some villagers who were in contact with infected animals. Deaths were not investigated, however, the villagers mentioned to visitor of the attacked village. This research is the first to mention that LSDV can infects human and exhibits skin lesions of various degrees, with primary localized nodules in upper of thigh, buttock, perianal areas, face, hands, with some mortalities [not investigated].

In this research, co-infection of herpes virus and LSDV was observed. Typical herpes virus particles were detected by DEM-NS on

${ }^{\star}$ Correspondence to: Samia Ahmed Kamal, Animal Health Research Institute (AHRI), Dokki, Giza, Egypt, E-mail: selkabany@yahoo.com

Key words: Lsdvh, lumpy skin disease in man, poxvirus zoonosis, lumpy skin disease, viral skin disease, bee venom, ascorbic acid, skin nodular disease

Received: December 05, 2019; Accepted: December 12, 2019; Published: December 16, 2019 
vesicle fluid, as the fragile envelopes of herpes viruses were penetrated by PTA stain - and reveals the stable virions cores. However, Herpes simplex is caused by two closely related viruses. Herpes Simplex Virus (HSV) type 1 and is mostly associated with oral cold sores and HSV2 is found in genital lesions [11-14]. Lumpy skin disease in cattle (main host), is distinguished - by high morbidity, higher contagious spreading, cutaneous lumps, fever, lymphadenopathy, emaciation, and an illness that may persist for months in animals. Mortalities are high in young animals, and all age groups showed mortalities specially in naïve herds. Man infections with LSD are to great extent resemble the illness pathogenesis in cattle, neglected cases in human may lead to complications, as systemic infections probably happen and expected in immune compromised persons. The disease in man would studied extensively in the future [15-18].

\section{Materials and methods}

1-Biological specimens: Blood samples and infected skin specimens were collected from infected persons (10). The preliminary identification was performed by negative staining by PTA (Phosphotungestic Acid) for Transmission Electron Microscopy (TEM). The procedures were performed according to Payment, et al. $[8,9,10]$.

2-Isolation of LSDVh: Isolation of LSDV from human samples were performed using BHK-21 [baby hamster kidney cells], and optimum temperature $38{ }^{\circ} \mathrm{C}$, according to Payment, et al. [8]. The isolates were kept in -80 deep freezing and given specific abbreviations; [LSDVh, 2018].

3- Convalescent anti-LSDVh positive sera: Sera samples were collected from some convalescent human subjects who completely recovered from LSDVH infections. These sera were tested by reference LSDV and showed satisfied titer of antibodies. This positive sera were useful in rapid diagnosis of new cases of LSDVh.

4- Electron microscopic examinations; Transmission EM: The procedures were performed in Cairo University. Direct electron microscopic examination by negative staining (DEM-NS) is a rapid method to demonstrate poxvirus in original unpurified biological specimens and samples by direct negative staining. Test procedures: Direct electron microscopy (DEM): The original biological specimens were re-suspended in $50 \mu \mathrm{L}$ of phosphate-buffered saline (PBS) $\mathrm{pH}$ 7.2. One drop of the suspension was put on EM grid and submitted to negative staining technique with $2 \%$ potassium phosphotungstate (PTK) $\mathrm{pH}$ 6.4. The grids were examined and the viruses were documented in a Philips EM400-T electron microscope operating in $80 \mathrm{kV}$. All samples examined by NS-DEM. LSD virions has the characteristic morphology of poxviridae family; large, enveloped, pleomorphic, brick-shaped appearance with irregularly arranged, surface short filaments. The procedures performed $[9,10]$.

5-Polymerase Chain Reaction (PCR) Assays: The conventional method and partial genome sequencing for ORF103 gene: Performed in reference Egyptian laboratory for animal viruses [AHRI, Dokki, Egypt].

a-Extraction of genomic DNA: The supernatant of prepared scabs samples were collected for viral DNA extraction using a Viral DNA Extraction Kit, according to manufacturer instructions and then used as a template in PCR.

b-PCR amplification: The open reading frames (ORFs) of the ORF 103 gene were amplified via PCR from DNA extracted from original samples. Primers: Target ORF103 gene for 570bp - seq. [Forward 5' ATGTCTGATAAAAAATTATCTCG 3'] [Reverse 5'
ATCCATACCATCGTCGATAG 3']. PCR amplification proceeded with an initial denaturation step of $94^{\circ} \mathrm{C}$ for 5 min followed by 35 cycles at $94^{\circ} \mathrm{C}$ for $1 \mathrm{~min}, 52^{\circ} \mathrm{C}$ for $45 \mathrm{~s}, 72^{\circ} \mathrm{C}$ for $1 \mathrm{~min}$, and a final extension of $72^{\circ} \mathrm{C}$ for $10 \mathrm{~min}$. Amplicons were visualized via electrophoresis on a $1.0 \%$ agarose gel and documented with a gel documentation system.

c-Cloning and DNA sequencing: The PCR products of the ORF 103 genes were then cloned. Comparison of the isolated sequences with those of Poxviridae available in the GenBank database was performed using the online Basic Local Alignment Search Tool program. Sequence identities of nucleotides between the isolated and NCBI sequences were analyzed. The nucleotide sequences deduced were assembled into a multiple sequence alignment.

\section{Results}

\section{Nature of lumpy skin disease in human}

Epidemiology: Human infections with LSDV are recorded in this research. Infected persons could be discovered in any areas where infected animals were present, so that sporadic human infections are still present and could be observed all over the country. LSD Virus is very sensitive and inactivated by glacial acetic acid, and Bee Venom. Recurrence of infection takes place without new exposure to a source of infections. All human subjects are liable to infection, however the disease showed severe forms in subjects with poor hygienic conditions, young ages and the immune compromised. The nodules responds to treatment by acyclovir drug. But nodules eruptions did not stopped by this drugs and new nodules appears in the infected persons. Person to person transmission were recorded, with apparent severe course of illness among this group of people who lives in very poor hygienic conditions. Recent infections [November 2019] were seen in persons who are living in areas were animals offal are distributed (Al Sayida Zeinab district) in Cairo, where abattoir was present. Incubation periods were in a range of one - two weeks.

The skin affections persist for months with formation of new nodules adjacent to the old ones. The nodular lesions did not respond to treatment by antibiotics, but broad-spectrum antibiotic (ceftriaxone) was helpful in combating secondary bacterial infections and showed relief of edema and inflammations. In the same patient, some nodules were showing good prognosis and disappeared without leaving scars after treatment by the oral administration of Acyclovir 1gram, topical bee venom ointment conc. $0.1 \%$, and ascorbic acid $1 \%$ lotion. However, other skin nodules leave scars, and need months for regression. Isolation of LSDVh was achieved from patients with inflamed and active skin nodules, however the virus was detected in skin nodules and sera samples.

Clinical symptoms: First symptoms were fatigue, weight loss, fever and itching in areas were nodules would be erupting, some cases showed herpes lesions preceding the appearance of the skin nodules, these nodules were unique and never resembles abscesses (Figure 1). Symptoms of disease in some patients were aggressive, as body and legs were severely affected with numerous nodules and ulcerations. During the progress of LSD in human, the skin nodules were spreading all over the body. The painful skin lesions owned to the severe edema which developed inside the affected areas which characterize the disease in human [LSDVh].

High body temperature degrees estimated by $38.5-38.9{ }^{\circ} \mathrm{C}$, recurrence of fever occurs when new nodules appear and during its growing. Edema and swollen lymph nodes were recorded in all subjects 


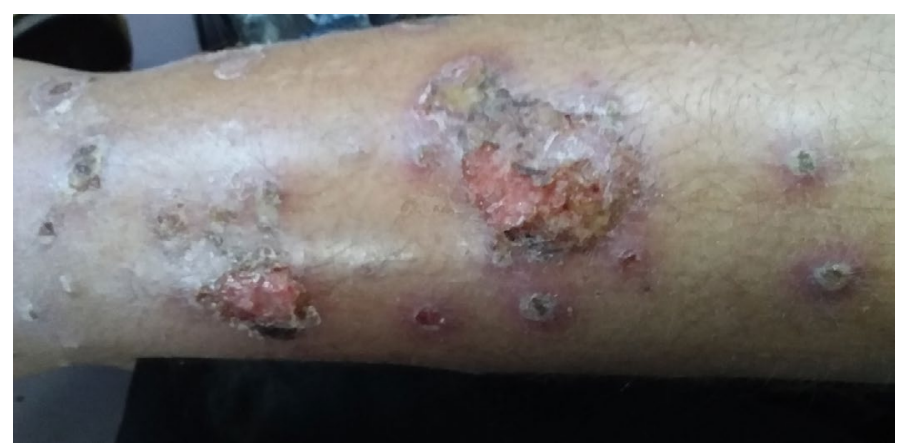

Figure 1. LSDV infection in leg of human patients, showing severe lesions [edema, ulcers, nodules]

whose showing skin nodules. Mode of skin eruptions seems to be variable from person to another, however nodules in all cases were erupted in the areas around genital organs. In farmers and abattoir personnel, skin nodules were mostly first seen in parts of body that handles infected materials, then were spreading to other sites in their bodies. However, some nodules developed firstly in skin of neck then spreads to other areas of body. Following fever, a sudden skin eruptions of painful, hard nodules were appear in certain and specific sites (hands, legs, face, genital organs, perianal regions, neck). Some cases showed eruptions of herpes virus in different parts of body, that followed by the development of large LSD nodules. The shape of LSD nodules in human were firstly appear as firm, red thickening occupy all layers of infected skin, with protruded apex and contain yellowish watery exudates that turned necrotic with the progress of disease. Since first lumps appear and occupying the entire thickness of the skin the ulcerations and formation of new nodules occurred then spreading the infections to other body parts.

\section{Electron microscope findings analysis}

\section{LSDV and Poxviridae family morphology detection}

Pox virus with characteristic morphology of capripoxviruses were detected in all examined samples (Figures 2-12). The negative stain-TEM is used in veterinary and medical virology diagnosis, with high degree of accuracy. This method allows rapid detection of poxvirus infection and very useful in differential diagnosis. DEM-NS are showing poxviruses as pleomorphic, brick-shaped, enveloped, large viruses that may reach $400 \mathrm{~nm}$ width. Some immature virions from aspirates of recently developed nodules were smaller in size, not enveloped and exhibiting clear short capsular filaments, however, both enveloped and non-enveloped virions are infectious. The process of poxvirus reproduction takes place inside cell cytoplasm, maturation and the addition of outer coat layers following virions release from its primary production site.

\section{Herpes viruses morphology}

DEM-NS (Figures 2-12) were showing the presence of poxviruses and herpes viruses. Herpes virions were visualized in different status of growth and replication, as the virions were enveloped with visible eccentric cores and/or non-enveloped forms. The herpes virus virions detected in the vesicular fluid were showing disintegrated envelopes contains eccentric cores that measures $45-52 \mathrm{~nm}$, adjacent to nonenvelope mature LSDV (Mulberry) $M$ form, indicating the functional activity of acute developing co-infection. The aggregations and mixing of various developmental stages of both herpes viruses and poxviruses inside same site indicates the functional co-infection (Figures 11 and 12).

\section{Results of conventional Polymerase Chain Reaction (PCR)}

Samples are positive for LSD virus by conventional PCR (Figure 13) and genetically characterized as Lumpy skin disease virus isolate Evros/ GR/15 by partial sequence with $99 \%$ identity. The virion contains a linear, A+T-rich, double-stranded DNA genome (Table 1).

Gene sequence target ORF103: Sequence target ORF 103 gene, showing the rich A-T nucleotides:

[....CTAATCCTTCAGCTACCACGTTTATTAA

CTTTTTTTTTGATTGTGAAATATTTTTGTTT

CGTCTACCAGCATTTACTGATTCTATTCCTA ACAAGTCTTGATTTATTTCACCTACCATACC TTGAACTTCTAATTTTCCTTCTTTCATGGTTC CGTATACTATTTTTCCGCCATTTGTTACTGCT TGCATTATTTGATCAGTTTCGTTAAATGTATT TCTTTTTTTAAAAACAGCTCCATTATTAACAC TTTTCGAAGAACATACGTTACCGCTTCTCGAA GTAGTTTGTTTATTACTGCAAGATTTTGATTT TTTATTTGGTTTTGTCATACAAGCTTTAGTTTT CACATTGCCAGCTTTTACTTCATTCTCATCTGT ATTATTAGTAGTATTATCCTCTGGAGTAAGATT TGATTTTTGAGATGCTTGTTCTCCACTAATGTG GGCAAGAATGGTCCTTAGTTG AGGGGTTAGTT TATTTATAGTTTCGATATAATCATCATAACTGC TTCGAGATAATTTT TATTAAGACAT....].

Reverse sequence [ORF103 gene]: Reverse sequence target ORF 103 gene, showing complementary rich A-T nucleotides.

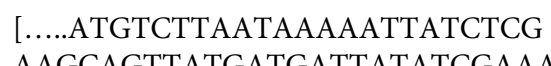
AAGCAGTTATGATGATTATATCGAAA

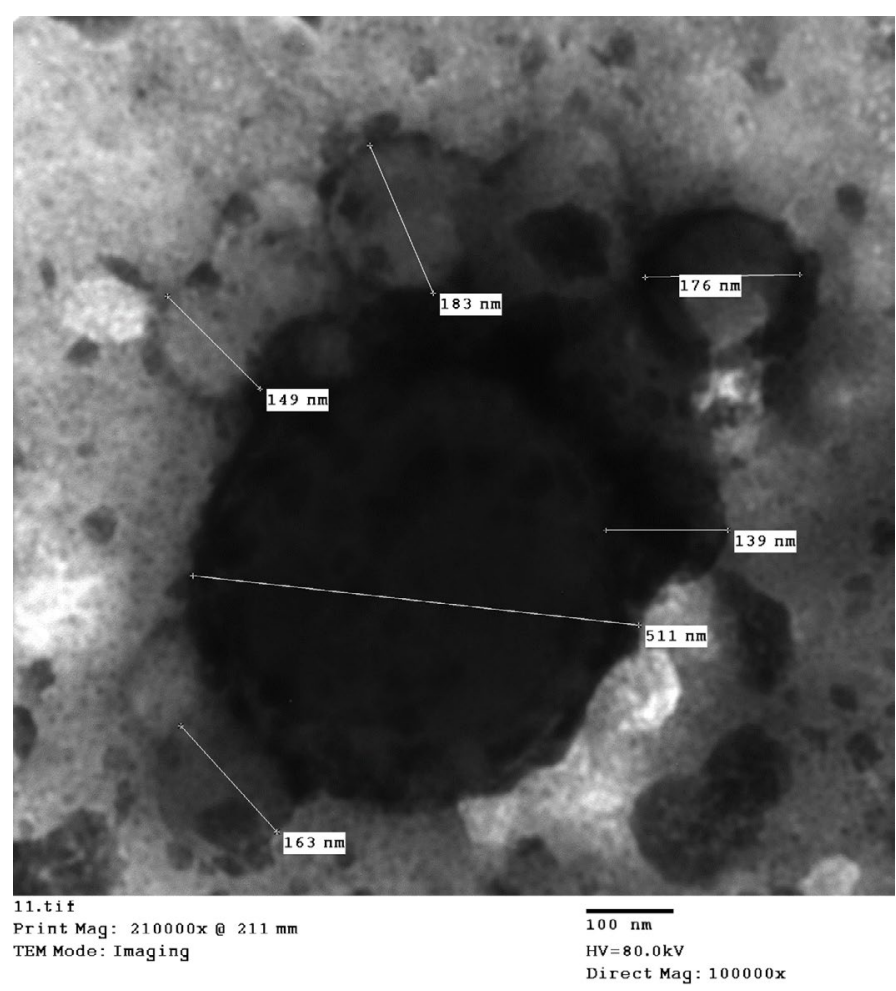

Figure 2. Human LSDV infections, skin lesion vesicular exudates showing, brick-shape virions [139-183nm in diameter]. These viral particles aggregates around an electron dense larger molecule $[511 \mathrm{~nm}$, width], and showing the $\mathrm{M}$ form and the textured surface structure of non-enveloped particle outer membrane. (Negative Staining, TEM). 


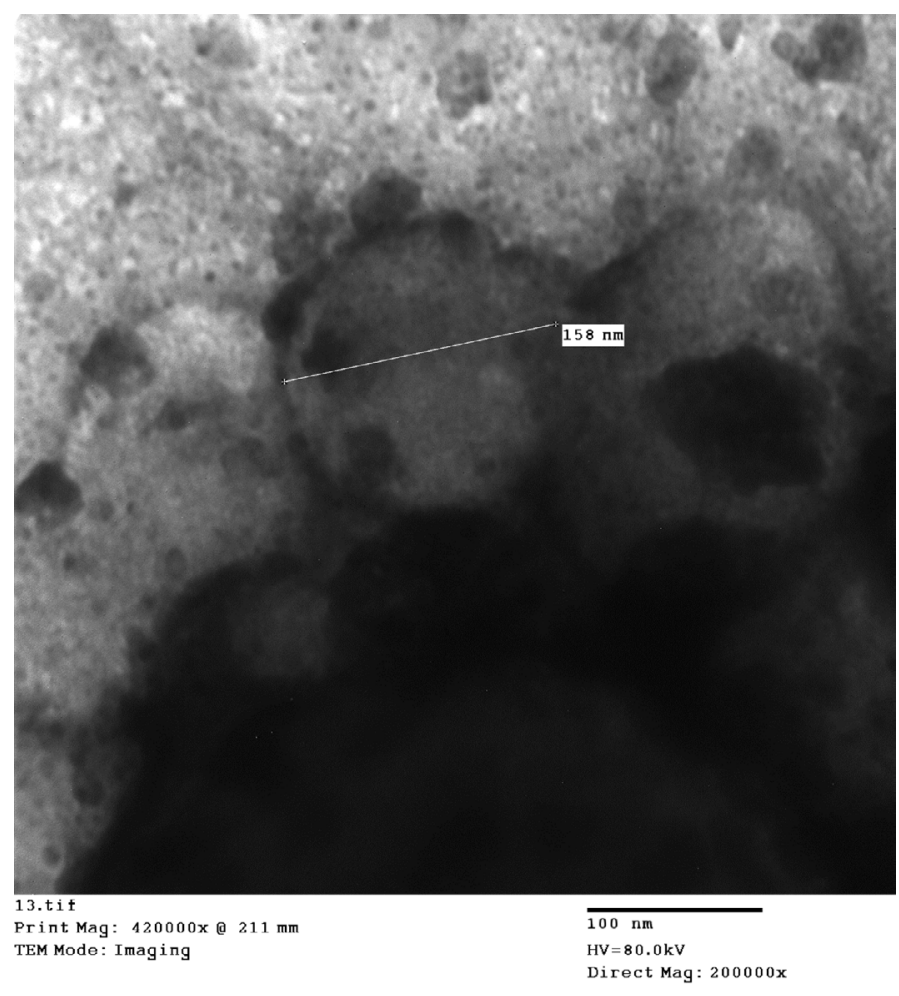

Figure 3. Human LSDV infections, skin vesicular fluids showing, Lumpy Skin Disease virions. the viral particles (158 nm width) aggregates around an electron dense larger molecule, showing the $\mathrm{M}$ form and the textured surface structure of non-enveloped particle outer membrane and is found mainly in the vesicular fluid (Negative Staining, TEM).

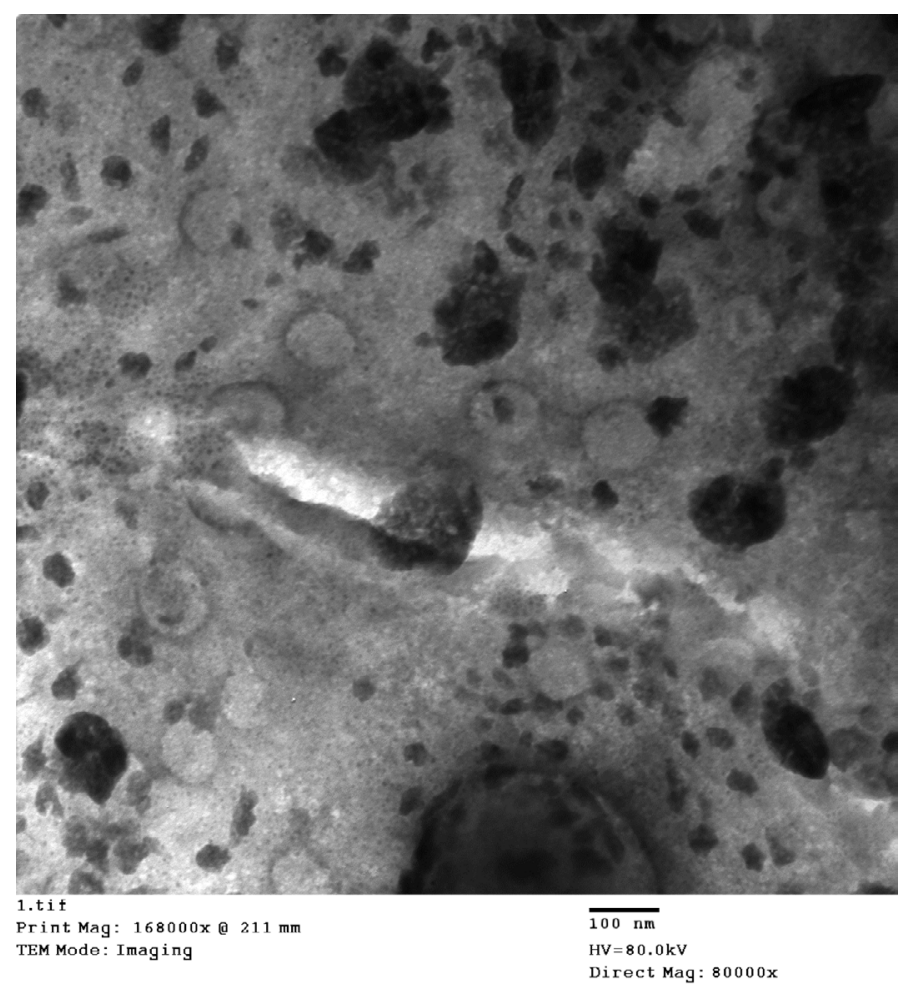

Figure 4. Human LSDV infections, skin vesicular fluids showing, Lumpy Skin Disease virions. showing the $\mathrm{M}$ form and the textured surface structure of non-enveloped particle outer membrane and is found mainly in the vesicular fluid (Negative Staining, TEM).

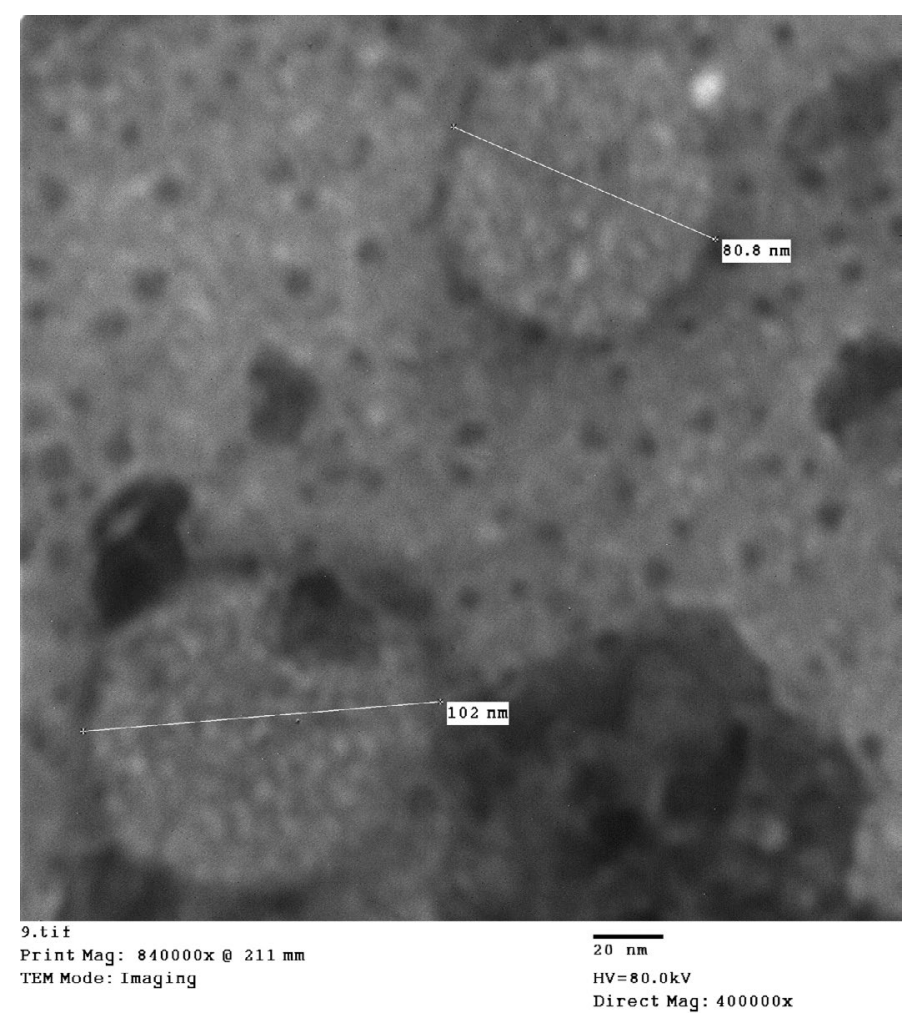

Figure 5. Human LSDV infections, skin vesicular fluids showing, Lumpy Skin Disease, viral showing the $\mathrm{M}$ form and the textured surface structure of non-enveloped particle outer membrane and is found mainly in the vesicular fluid (Negative Staining, TEM).

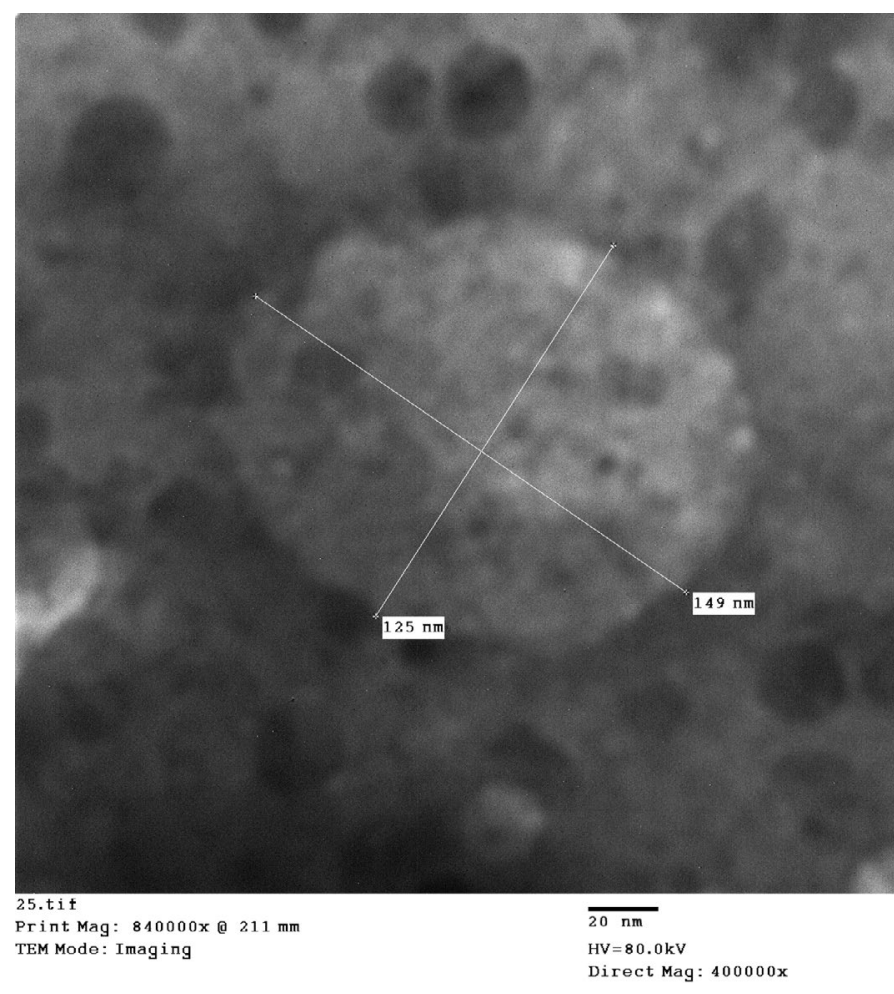

Figure 6. Human LSDV infections, skin lesion vesicular fluid showing: large, nonenveloped virion [125 nm x 149nm], with characteristic morphology of pox virus; brickshaped, with short surface short tubules (Negative Staining, TEM). 


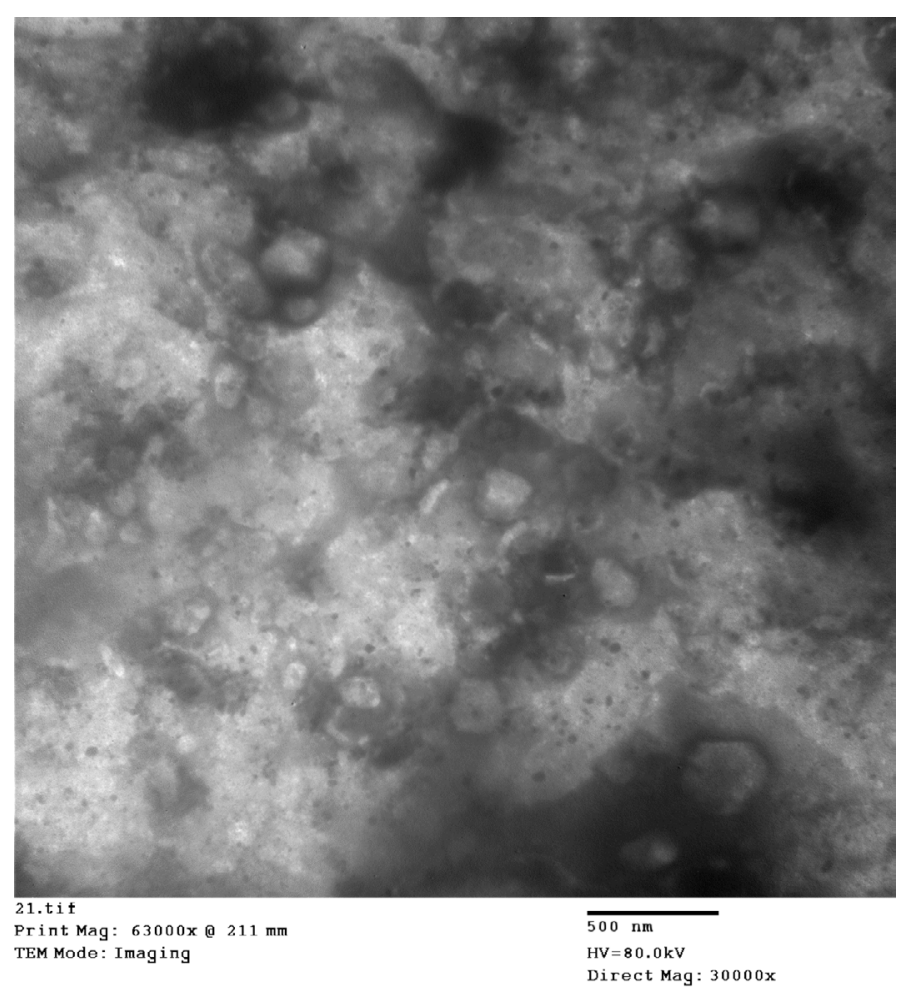

Figure 7. Human LSDV infections, skin lesion vesicular fluid showing; virions are brick shaped, enveloped, pleomorphic with smooth envelope (Negative Staining, TEM).

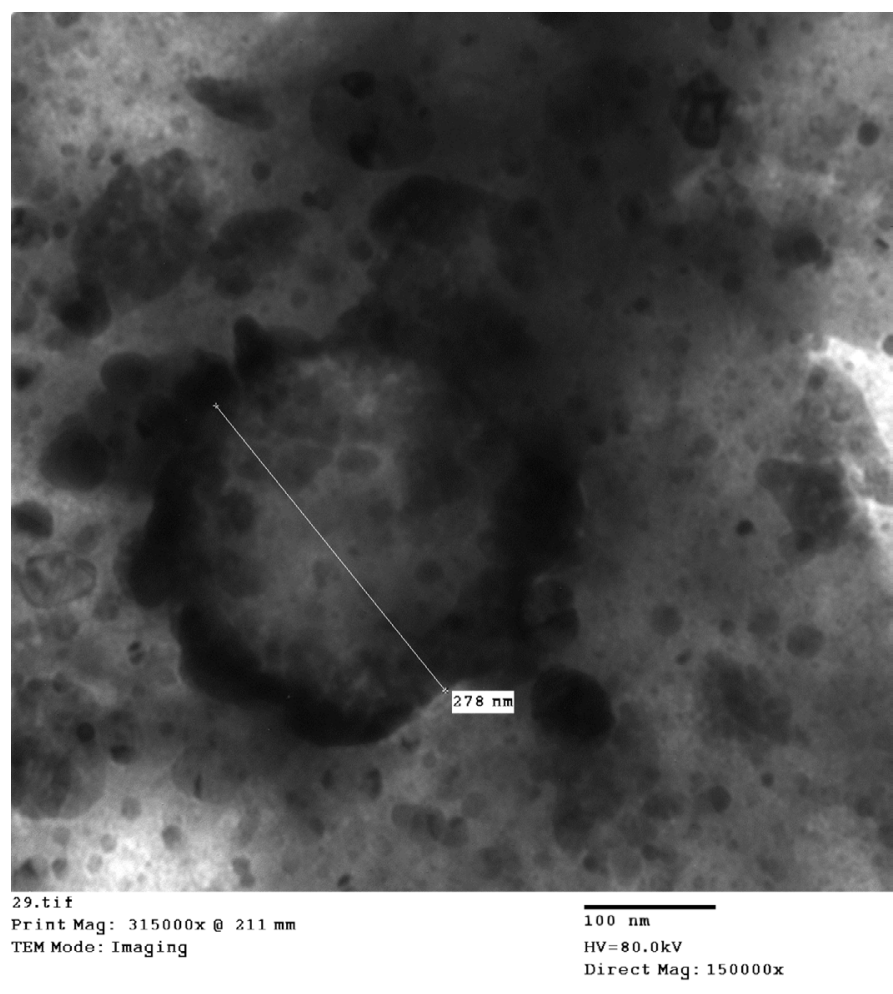

Figure 8. Human LSDV infections, skin lesion specimen showing; virions are brickshaped, mature and enveloped [167nm-344 nm], pleomorphic with smooth envelope (Negative Staining, TEM).

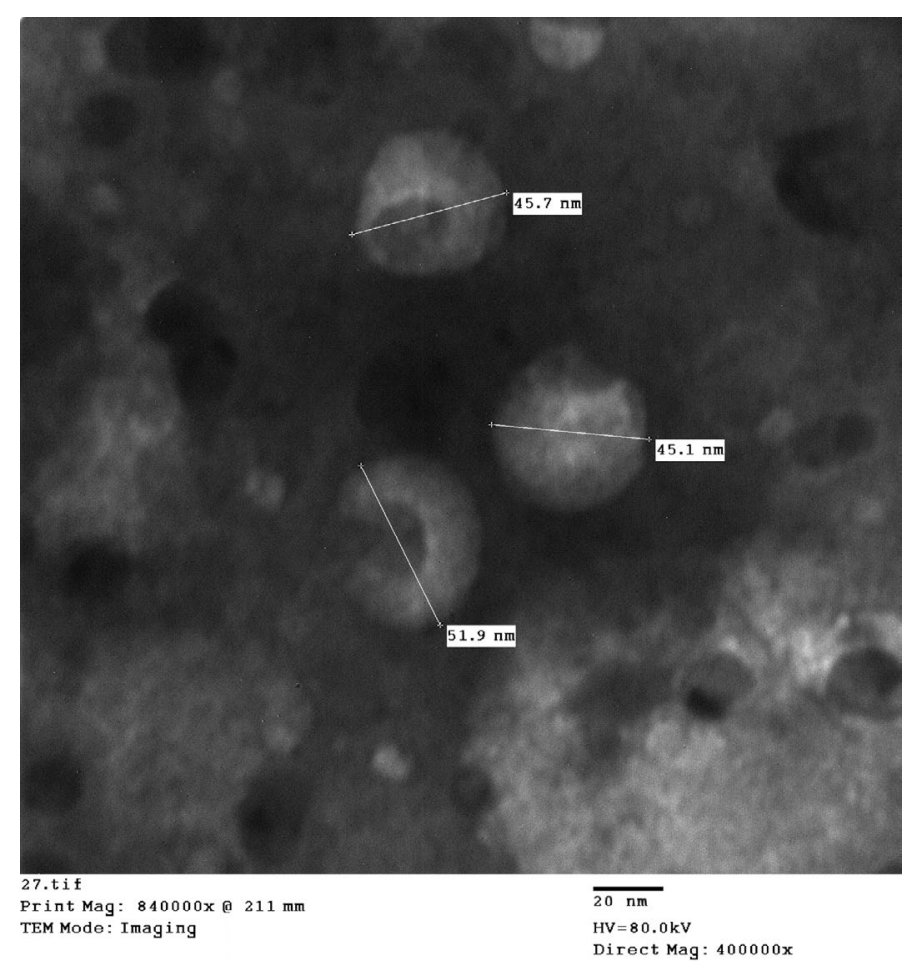

Figure 9. Human herpes virus, vesicular fluid showing: disintegrated envelopes and herpe viruses cores measures [ $45 \mathrm{~nm}-52 \mathrm{~nm}$ ], along with $\mathrm{M}$ form of LSDV [non-envelope], indicating the functional activity of acute developing co-infection. (Negative Staining, TEM).

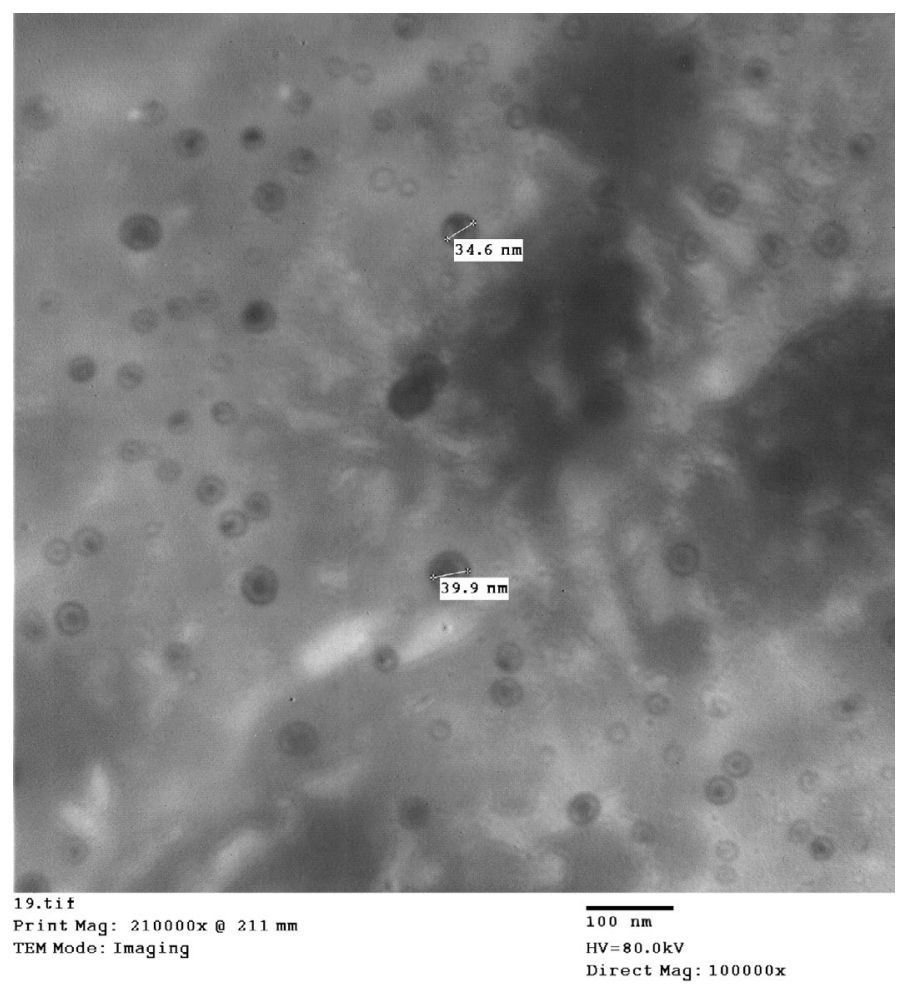

Figure 10. Human herpes virus, vesicular fluid showing: naked herpes viruses measures [34 nm - $40 \mathrm{~nm}$ ], indicating the functional activity of acute developing co-infection. (Negative Staining, TEM) 


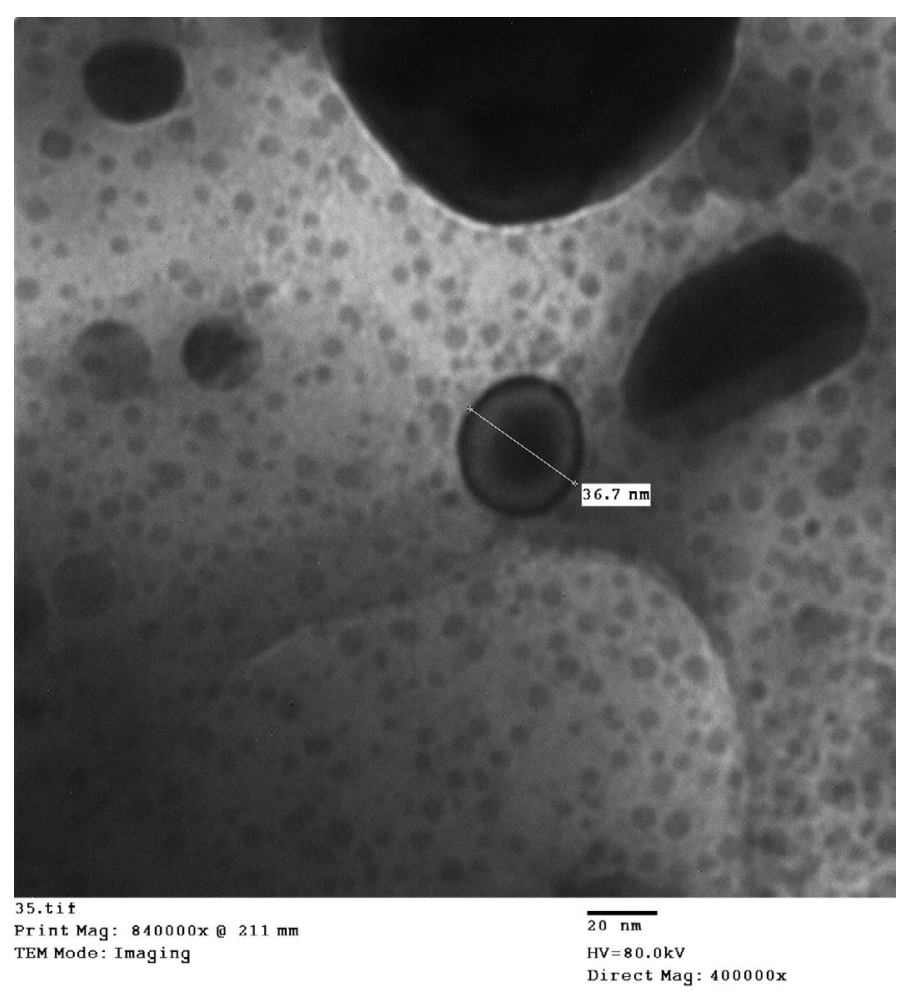

Figure 11. Human herpes virus, vesicular fluid showing typical morphology of HHSV. The naked virion measures [38 nm width] (Negative Staining, TEM).

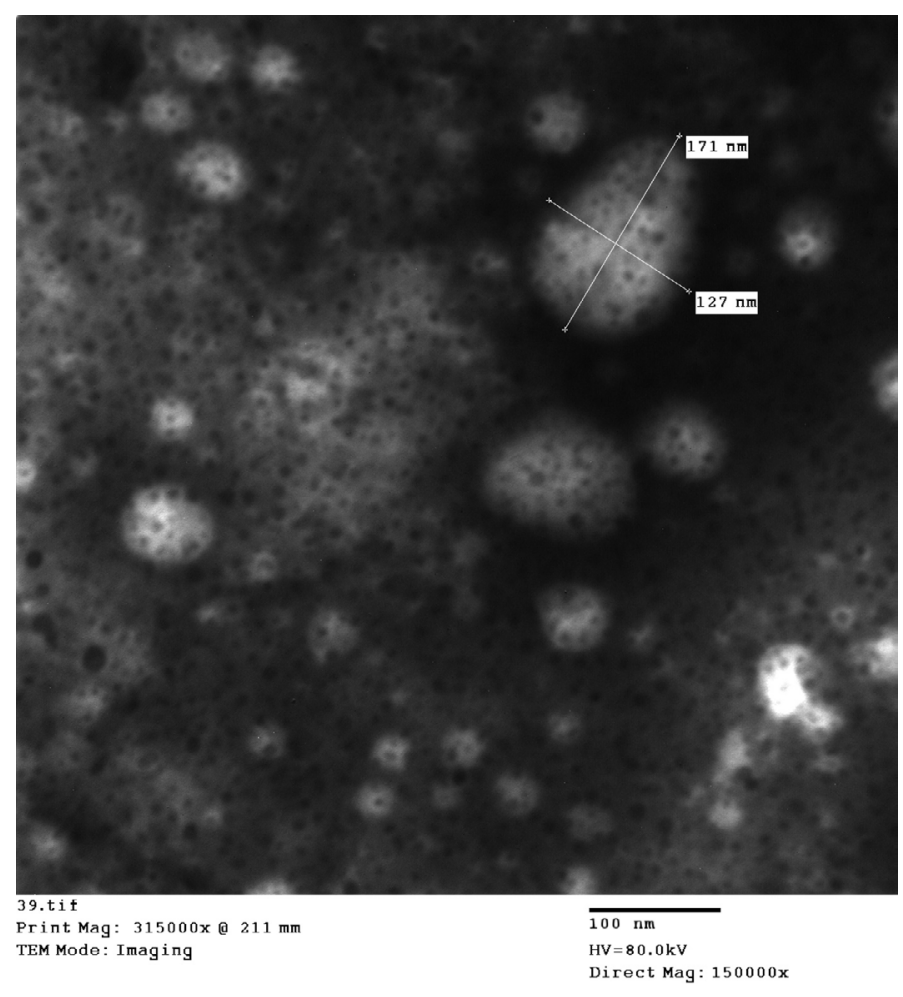

Figure 12. Human LSDV infection, skin lesion specimen showing some virions of LSDV and Herpes viruses; virions of pox virus are enveloped oval of [171 nm x $127 \mathrm{~nm}$ ], or brick-shaped, and mature with pleomorphic smooth envelope. Herpes viruses also seen in between the pox viruses virions (Negative Staining, TEM).

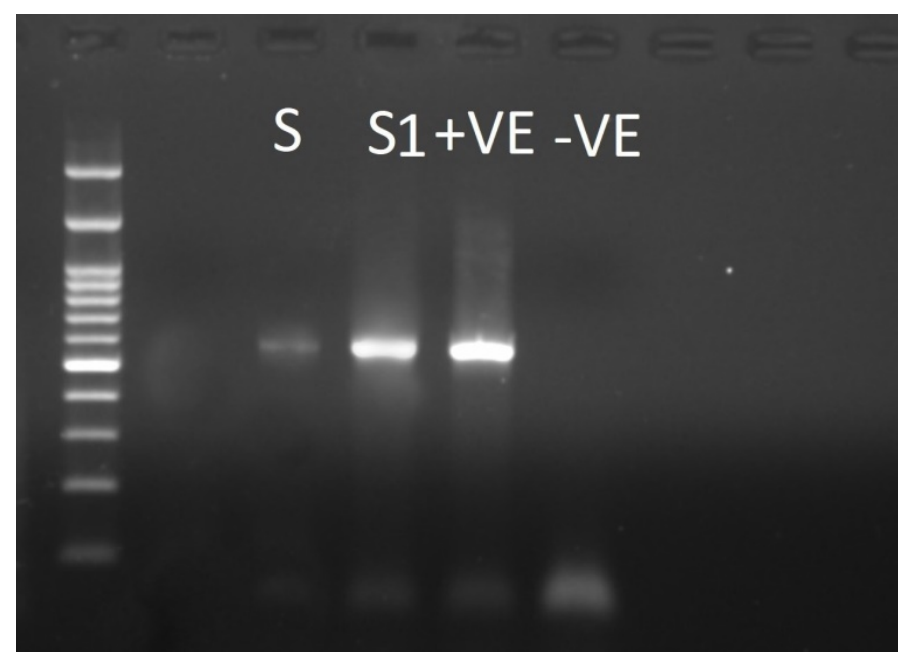

Figure 13. PCR showing positive results of LSDVh

CTATAAATAAACTAACCCCTCAACTAA GGACCATTCTTGCCCACATTAGTGGAG AACAAGCATCTCAAAAATCAAATCTTA CTCCAGAGGATAATACTACTAATAATA CAGATGAGAATGAAGTAAAAGCTGGCA ATGTGAAAACTAAAGCTTGTATGACAAA ACCAAATAAAAAATCAAAATCTTGCAGT AATAAACAAACTACTTCGAGAAGCGGTA ACGTATGTTCTTCGAAAAGTGTTAATAAT GGAGCTGTTTTTAAAAAAAGAAATACATT TAACGAAACTGATCAAATAATGCAAGCAG TAACAAATGGCGGAAAAATAGTATACGGA ACCATGAAAGAAGGAAAATTAGAAGTTCA AGGTATGGTAGGTGAAATAAATCAAGACTT GTTAGGAATAGAATCAGTAAATGCTGGTAG ACGAAACAAAAATATTTCACAATCAAAAAAA AAGTTAATAAACGTGGTAGCTGAAGGATTAG.....

\section{Discussion}

The present investigation has discovered that LSD virus can infect human as zoonotic disease, and showing skin affections resembling that of cattle. Modes of transmission are by direct contact with infected cattle, infected utensils, aerosol, laboratory acquired infections and person to person transmission, and apparently have a highly contagious nature in man. Persons in contact with infected cattle were easily and simply catch LSDV infection. Samples from human are positive for LSD virus by conventional PCR (Figure 13) and genetically characterized as Lumpy skin disease virus isolate Evros/GR/15 by partial sequence with $99 \%$ identity (Table 1). The partial sequencing by ORF103 showing that human was infected with the same strain circulating in that outbreak (Table 1). The virion contains a linear, A+T-rich, double stranded DNA genome. The pox virus virion has more complex set of polypeptides, that have arranged in four distinct structures (core, lateral body, membrane, and envelope) which observed by the electron microscope. Pox viruses membranes are lipoprotein bilayer that surrounds virions cores. The outer surface is studded with surface short tubular units, showing the pox virus virion its textured surface. The virion composed of a nucleoprotein core and associated lateral bodies surrounded by the membrane, is sufficient for infectivity, and some 
Table 1. Sequence alignments target ORF103

Sequences producing significant alignments

\begin{tabular}{|c|c|c|c|c|c|c|}
\hline Description & Max score & Total score & Query cover & E value & Ident & Accession \\
\hline Lumpy skin disease virus isolate $155920 / 2012$. complete genome & 939 & 939 & $97 \%$ & 0.0 & $99 \%$ & KX894508.1 \\
\hline Lumpy skin disease virus isolate Evros/GR/15. complete genome & 939 & 939 & $97 \%$ & 0.0 & $99 \%$ & KY829023.3 \\
\hline Lumpy skin disease virus isolate SERBIA/Bujanovac/2016. complete genome & 939 & 939 & $97 \%$ & 0.0 & $99 \%$ & KY702007.1 \\
\hline Lumpy skin disease virus NW-LW isolate Neethling Warmbaths LW. complete genome & 939 & 939 & $97 \%$ & 0.0 & $99 \%$ & AF409137.1 \\
\hline Lumpy skin disease virus strain KSGP 0240. complete genome & 928 & 928 & $97 \%$ & 0.0 & $99 \%$ & KX683219.1 \\
\hline Lumpy skin disease virus NL-2490 isolate Neethling 2490. complete genome & 928 & 928 & $97 \%$ & 0.0 & $99 \%$ & AF325528.1 \\
\hline Lumpy skin disease virus isolate Cro2016. complete genome & 905 & 905 & $97 \%$ & 0.0 & $98 \%$ & MG972412.1 \\
\hline Lumpy skin disease virus strain neethlling-LSD vaccine-OBP. complete genome & 905 & 905 & $97 \%$ & 0.0 & $98 \%$ & KX764645.1 \\
\hline Lumpy skin disease virus strain neethlling - Herbivac vaccine. complete genome & 905 & 905 & $97 \%$ & 0.0 & $98 \%$ & KX764644.1 \\
\hline Lumpy skin disease virus strain SIS-Lumpyvax vaccine. complete genome & 905 & 905 & $97 \%$ & 0.0 & $98 \%$ & KX764643.1 \\
\hline Lumpy skin disease virus isolate Neethling vaccine LW 1959. complete genome & 905 & 905 & $97 \%$ & 0.0 & $98 \%$ & AF409138.1 \\
\hline
\end{tabular}

virions acquires the envelope as additional lipid bilayer that showing the pox viruses as pleomorphic and smooth brick shaped appearance (Figures 7,8,12). Negative staining- direct electron microscopic (NSDEM), was invented by Brenner \& Horne in 1959, the technique based on the Phospho-tungstic acid [PTA] as an electron opaque substance is absorbed by some biological substances. The image formation is the result of electron being absorbed or deflected by the stain $[7,10]$.

The Herpes virus mature virions are enveloped, ds-DNA genome, with specific TEM morphology. A peculiar feature of human Herpes Simplex (HHS) and Mareks disease virus DNA is that they fragment into several pieces of definite size when centrifuged on alkaline sacrose gradients. Herpes simplex virus, under certain conditions, has been shown to have cytotoxic effects, both in vivo and in vitro, which are separable from productive infection [12]. Some experimental studies found that heat-inactivated virus had a pyrogenic effect in guinea pigs. This effect could be partially neutralized by antiserum [19]. This specific observation is very useful in control efforts of herpes virus by vaccination, as the old and classic manner of vaccine preparations are not suitable to herpes viruses. Finding antiviral medicine is the most effective way to control major pathogenic agents inside Herpesviridae family

The outstanding presentations of herpes virions side by side with LSDV virions, showing the evidence of its importance in bringing about the full result of the disease (Figure 10). Which means that herpes virus infection is a helpful factor in the incidence of pox viral disease. Also the presence of viruses that cause skin damage and multiply in the same cells indicates their cooperation and integration, but perhaps the exchange of important elements during reproduction. That findings showing the intimate relation between herpes virus and poxvirus; both are DNA viruses and both predilection sites are the epithelium. It could be postulated that poxviruses able to exerts their effect by the presence of the indispensible herpes viruses co-infections. So that, these findings may help in our fights against diseases of poxvirus in man and animals, as the treatment of herpes virus infections by antiviral medicine or green disinfectant which are able to kill herpes virus will stop the progress of poxvirus skin lesions. These findings are in accordance with the rapid and successful prognostic effect following application of ascorbic acid lotions on skin lesions of persons infected with LSDV. This point need more investigation for unveiling the precise role of herpes viruses in the pathogenesis of poxviruses. Herpes viruses are ubiquitous in nature, that cause a wide variety of diseases. Two types of human herpes simplex virus [HHSV] fully elucidated: HSV-1 is usually associated with Oro-Facial disease, and HSV-2 is associated with genital disease. Lesion location, however, is not necessarily indicative of viral type, as HSV-1 is associated with genital infections more often than
HSV-2. Dendritic cells are antigen presenting cells that are found in the skin, at mucosal surfaces and in the blood. Their role is to survey the environment for pathogens, take up and process antigens and present antigens to T lymphocytes [11]. The electron microscopic studies on the morphology of herpes viruses, the negative staining method of Brenner \& Horne (1956) revealed the morphological detail which has become so characteristic of the group [10]. Epstein (1962a) has described the envelope as a triple-layered, membranous structure indistinguishable from host cell membrane [13]. Epstein (1962b) showed that the dense core region contained DNA and type of fixation and embedding affected core morphology [14]. Herpes viruses and LSDV co-infection was an outstanding findings in both forms of LSD [Cattle, and human]. Aggregations of various developmental stages of both herpes viruses and poxviruses, the outstanding mixing indicates the functional coinfection and synergistic interaction However; recovery from infections with herpes viruses, unlike recovery from infections with many other viruses, is not associated with complete immunity. The lesions may be caused by a chronic infection which is periodically activated, or the lesions may result from reinfection by virus from exogenous sources [4,11].

The approximate size of the virus may be determined by the stages of virus formation within the cell. Similarity was observed in the shape and the difference in size. It have noticed many forms of the virus, which may change as a result of the functional condition of the virus or the treatment of the immune system or its biological and synthetic condition. Shapes may affected by the method of preparing samples, or the effect of electrons and dye, all hypotheses make us not make what we see something absolutely certain and build a lot on it. So we should write what we observed as it is, maybe we cannot explain it at the same moment but it will have good results in the future. The form of the virus may reflect the nature of its biological function and its style in attacking different types of body cells as well as changes in the functional characteristics of the virus. The results obtained should therefore be recorded. However unmatched.

Human infections were following the handling of lumpy skin disease infected cattle [widespread outbreak, in 2018, Egypt]. Persons in contact and butchers were infected with skin nodules at perianal, buttock, skin of genital organs, face and hands. Some severe infections were mentioned by public with absence of health supervisions; two people at least were died during the outbreak in Al-fayoum Governorate. However, infected persons showing deep and painful cutaneous nodules (lumps) in face, hands and genitalia. Many locations of Egypt during year 2018, showed widespread outbreaks with sporadic human infections. It seems good that the people in the areas of LSDV outbreaks abstain from eating the meat of cattle, even the fish because dead cattle thrown into water channels. 
In human the LSD virus are very dangerous if affecting skin adjacent to vital organs like eyes, because this disease does not stop at a certain point. It was observed that after recovering and disappearance of LSDVh skin nodules and the wound dries up, another area adjacent to the first showed new nodules, mostly in skin were herpes virus usually seen and wherever humidity and warmth. The casualty of cattle traders and Bedouin usually have to deal with the injuries of their hands by thermo-catering. The disease lasts for months and does not leave the patient for several months despite receiving the supportive treatments. The similarities of skin nodules are consistent with what has been observed in humans as a result of cow pox and Orf viruses zoonosis [7].

However, the rural environment in Egypt, harbor huge number of live and active viruses because of recent poxviruses outbreaks along with other viruses strains which circulates naturally. As is now the use of serious viruses [cowpox virus] in pharmaceutical products [as immune modulators in horse] especially in veterinary field, where restrictions is weak in comparisons with human medicine. These biological drugs are dangerous and lead to the spread of these viruses. Moreover, members of the poxvirus family should be treated with the utmost caution.

Therefore, studies should focus on the discovery of antiviral drugs. However, meat of infected cattle is not suitable for human consumptions and the infected cattle should culled.

Some remedies can relief the affected skin. In the field conditions, it was useful to use glacial acetic acid and ascorbic acid for eradicating the skin nodules and killing the virus. However, to control LSDVh generalized infection, acyclovir and the Apis mellifera L venom (Bee venom) are recommended $[20,21]$.

\section{Conclusions}

This work discover the LSDVh for the first time in history. The LSDVh was isolated on BHK-21 successfully. The sickness of Lumpy skin disease in human was described and the diagnosis was confirmed by NS-DEM and the Conventional Polymerase Chain Reaction and partially sequencing using the ORF103 gene. In this research, coinfection of herpes virus and LSDVh has observed.

\section{References}

1. Prozesky L, Barnard BJ (1982) A study of the pathology of lumpy skin disease in cattle. Onderstepoort J Vet Res 49: 167-175. [Crossref]

2. Samia Ahmed Kamal (2016) In Vitro Study on the Effect of Bee Venom on Some Cel Lines and Lumpy Skin Disease Virus. Journal of Agricultural Science and Technology 6: $124-135$.
3. Tuppurainen ES, Oura CA (2012) Review: lumpy skin disease: an emerging threat to Europe, the Middle East and Asia. Transbound Emerg Dis 59: 40-48. [Crossref]

4. House JA, Wilson TM, el Nakashly S, Karim IA, Ismail I, et al. (1990) The isolation of lumpy skin disease virus and bovine herpesvirus-4 from cattle in Egypt. J Vet Diagn Invest 2: 111-115. [Crossref]

5. European Commission (2016) concerning animal health control measures relating to lumpy skin disease in certain Member States. Official Journal of the European Union L 310: 51-65.

6. Carn VM, Kitching RP (2015) An investigation of possible routes of transmission of lumpy skin disease virus (Neethling). Epidemiol Infect 114: 219-226. [Crossref]

7. Rodhain F (2015) Insects as vectors: systematics and biology. Rev Sci Tech 34: 83-96, 67-82. [Crossref]

8. Mark R, Buller L, Gregory JP (1991) Poxvirus Pathogenesis. American Society for Microbiology 55: 0146-0749.

9. Payment P, Trudel M (1993) "Methods and Techniques in virology." Text book Marcel Dekker INC.

10. Hayat MA (1981) Principles and techniques of electron microscopy. Biological applications, second ed Vol. 1.

11. Brenner S, Horne RW (1959) A negative staining method for high resolution electron microscopy of viruses. Biochim Biophys Acta 34: 103-110. [Crossref]

12. Kaplan AS (1973) The Herpesviruses. Text book. Academic Press INC, USA.

13. Lee LF, Kieff ED, Bachenheimer SL, Roizman B, Spear PG, et al. (1971) Size and composition of Marek's disease virus deoxyribonucleic acid. J Virol 7: 289-294. [Crossref]

14. Epstein MA (1962) Observations on the mode of release of herpes virus from infected hella cells. J Cell Biol 12: 589-597. [Crossref]

15. Epstein MA (1962) Observations on the fine structure of mature herpes simplex virus and on the composition of its nucleoid. J Exptl Med 115: 1-12. [Crossref]

16. Gari G, Waret-Szkuta A, Grosbois V, Jacquiet P, Roger F (2010) Risk factors associated with observed clinical lumpy skin disease in Ethiopia. Epidemiology and Infection 138: 1657-1666. [Crossref]

17. Tuppurainen E, Alexandrov T, Beltra'n-Alcrudo D (2017) Lumpy skin disease: a field manual for veterinarians. FAO Animal Production and Health Manual No. 20.

18. Dougherty CT, Knapp FW, Burrus PB, Willis DC, Burg JG, et al. (1995) Stable flies (Stomoxys calcitrans L.) and the behavior of grazing beef cattle. Appl Anim Behav Sci 42: 231-248.

19. Chihota CM, Rennie LF, Kitching RP, Mellor PS (2001) Mechanical transmission of lumpy skin disease virus by Aedes aegypti (Diptera: Culicidae). Epidemiol Infect 126: 317-321. [Crossref]

20. Tokumaru T (1963) Hypersensitivity to herpes simplex in guinea pigs. Federation of American Societies for Experimental Biology Proceeding 22: 616.

21. Samia Ahmed Kamal (2018) A New Discovery on How Bee Venom Components Treat Viruses. An Innovative Study on Camel Pox. SF Virol Res J 2: 1.

Copyright: (C2019 Kamal SA. This is an open-access article distributed under the terms of the Creative Commons Attribution License, which permits unrestricted use, distribution, and reproduction in any medium, provided the original author and source are credited. 